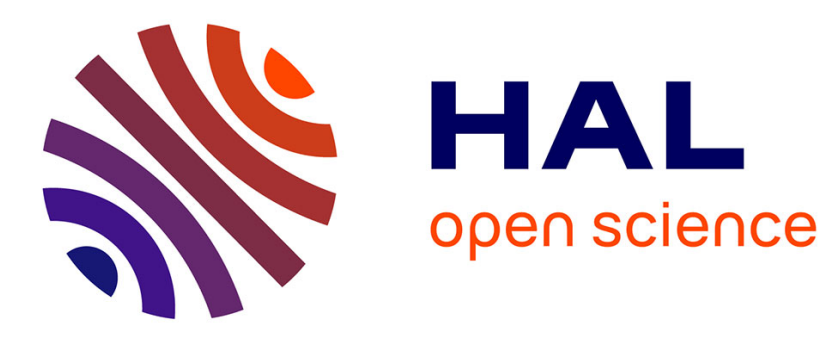

\title{
Moules de vases sigillés trouvés à Barzan (Charente-Maritime) \\ Hugues Vertet
}

\section{To cite this version:}

Hugues Vertet. Moules de vases sigillés trouvés à Barzan (Charente-Maritime). Gallia - Fouilles et monuments archéologiques en France métropolitaine, 1962, 20 (2), pp.412-418. 10.3406/galia.1962.2365 . hal-01926345

\section{HAL Id: hal-01926345 \\ https://hal.science/hal-01926345}

Submitted on 25 Feb 2020

HAL is a multi-disciplinary open access archive for the deposit and dissemination of scientific research documents, whether they are published or not. The documents may come from teaching and research institutions in France or abroad, or from public or private research centers.
L'archive ouverte pluridisciplinaire HAL, est destinée au dépôt et à la diffusion de documents scientifiques de niveau recherche, publiés ou non, émanant des établissements d'enseignement et de recherche français ou étrangers, des laboratoires publics ou privés.

\section{(이) $\$$}

Distributed under a Creative Commons Attribution - NonCommercial - NoDerivatives $\mid 4.0$ 
sommes réduits à l'analyse interne. Il paraît, certain que la frise représente une pompa et un sacrifice qui se déroulent en pleine campagne: les arbres qui peuplent le fond en sont la preuve. Certain, également, qu'il s'agit d'une cérémonie municipale : deux licteurs ouvrent le cortège. Trìs probablement, la pompa faisait le tour de l'autel, ce qui explique la position centrale de celui-ci. On a donc: affaire à une lustration. A quelle divinilé s'adressait ce culte rustique et municipal a la fois, où le sacrifice semble bien précédé d'une lustration? A Cérès? I Tellus? Enfin, le sacrifice consiste en suovélauriles. Faut-il y voir l'offrande normale a la déesse qu'on voit assise pres de l'autel? Ou bien, comme nous le croyons, ces suovétauriles sont-ils le sacrifice de consécration de l'autel, et leur représentation sur la frise a-t-elle pour but de commémorer le moment originel du monument? Telle nous paraît être la problématique de la frise des suovétauriles de Beaujeu.

1. Audix el Paul Verne.

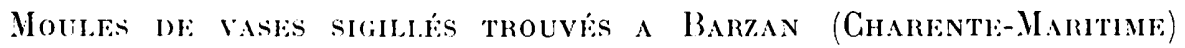

La pelite ville gallo-romaine du Fâ (le nom vient de Fanum), près de Tahmont, en CharenteMarilime, était alimentée par la grosse source de Chauvignac dont les eaux lui étaient amenées par un acfueduc de 3 kilomelres. Il s'agit en fail, ecrit M. Colles, professeur de géographie au college de loyan. d'une véritable riviere soulerraine qui apparait au bas des coteaux crétacés de Chenac (étage campinien), dans un creux profond ou " grourd". Elle est d'une grande purelé, car elle a été filtrée par les gres el les sables cenomaniens sousjacents'. Dès son apparition a l'air libre, elle forme aussitòt un ruisseau de :) mètres de large, qui, au bout de 1400 metres de parcours, va se jeter dans le chenal "des Monards"; puis dans la Gironde.

En 195\%, la source de Chauvignac a élé aménagée pour donner de l'eau aux communes avoisinantes. Au cours des installations de raptage, la fontaine a été curée. M. Colles. altentif aux antiquites de la région, a examiné les vases relirés. Il a pu recueillir des monnaies romaines de très belle conservation qui se réparlissent ainsi : 1 Vespasien. 1 Domilien,

(1) A. Plaxchet, I. Basalo, J.-R. Coldes, Le fia de Talmont, porl gallo-romain, Tours, 1944. -Louis Basalo, le temple du mollin du Fà à Barzan, press Talmonl-sur-Gironde, dans Gallia, II (1944), p. 141-165. - Gallia, Informations archéologiques, $\mathrm{XV}$ (1957), 2, p. 211-213. - Gallia, Informations archeologiques, XVII (1959), p. 476.
5 Trajan, 1 Hadrien. Mais, outre ces monnaies, qui n'ont pas encore été étudiées par un numismale. il a sauvé aussi des fragments de ceramique sigillée, el quatre morceaux de moules de vases à reliefs. Il a conservé soigneusement le toul el a "ul'amabilité de me le confier pour élude, ce dont je le remercie vivement : ces moules sont d'un intérêt exceptionnel.

\section{Moules.}

1) Iragment de moule de vase caréné, Dragendorfi 29) (pl. 1, no 1 el pl. 4. no 1). La terre, rose, assez peu cuile, peut d̀ re ravé a l'ongle et les décors sont devenus assez. frusles. Les parois sont asse\% minces : 6 millimetres; leur sommet assez aigu; le renforcement du col ne dépasse pas 9 millimètres d'épaisseur et il est bordé d'une rainure profonde. Il ne subsiste du décor de la panse qu'une parlie tres restreinte qui laisse supposer des rinceaux plut ol que des medaillons mais celui de la frise esl entier. - Entre les deux. courl la classigue moulure longée de deux lignes pointillés. comme sur le no 2 . La bande supérieure (hauleur $2 \mathrm{~cm} .33$ est ornée d'un rinceau dont les tiges filiformes ne portent pas de feuilles, mais seulement de toutes petites étoiles ou fleurelles, semées aussi dans le champ. La forme des ligalures des liges est celle qu'Oswall a donnée sous le no 19. Elle a élé relevée sur un vase signé of agcitani trouvé a l'indonissa et daté du 


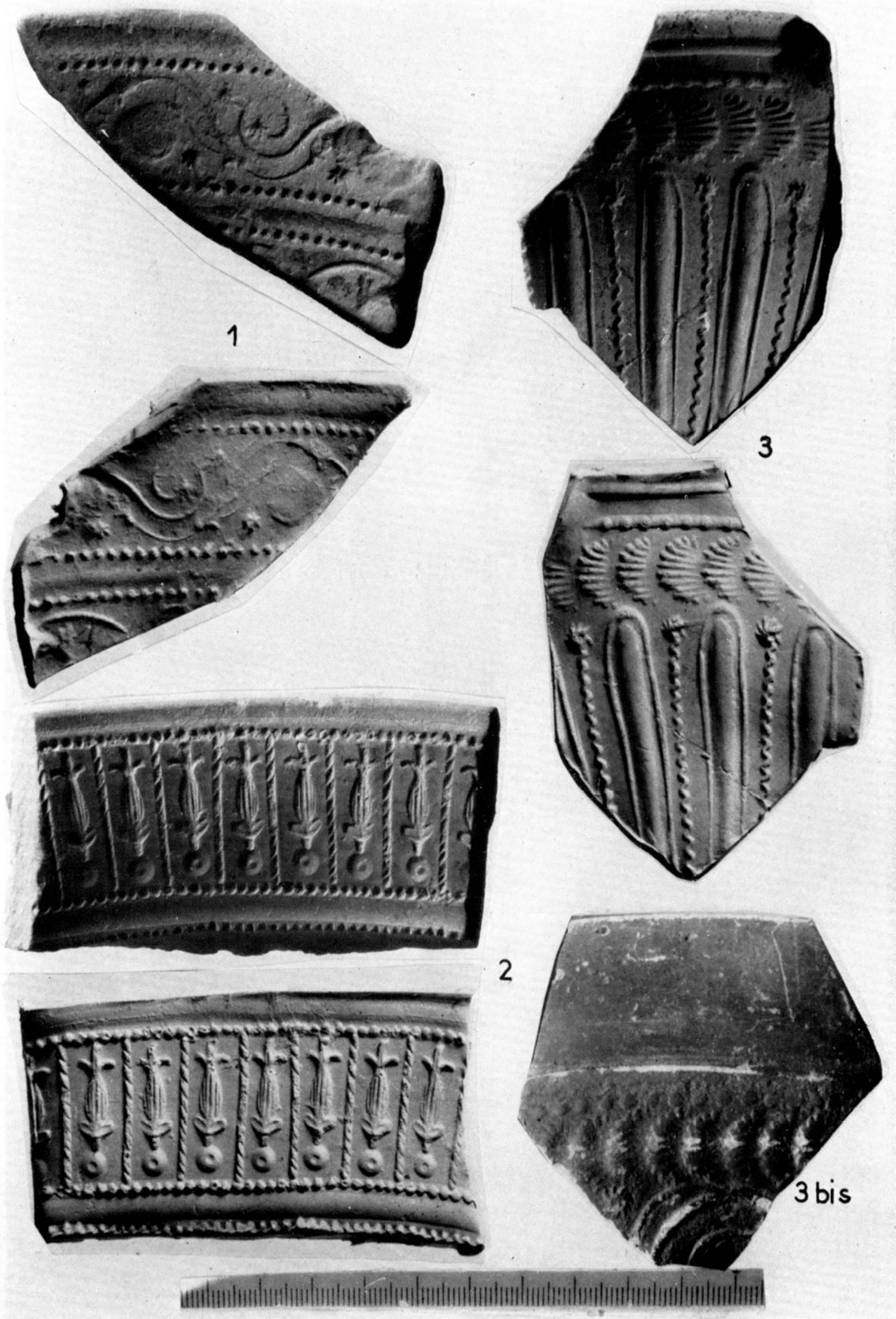

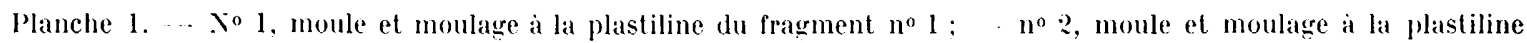
du fragment $n^{\circ}:$ : : - $n^{\circ} 3$, moule et moulage a la plastiline du fragrment $n^{\circ} 3 ; \ldots n^{\circ} 3$ bis, tesson du Musée de Périgueux, d'un vase de forme analogue à celle du moule no 3. (Pholi) $I$. Verlel). 
regne de Claude ${ }^{2}$. L'ensemble du décor, la forme peu évasée, la ténuité des dessins. confirment celle date pour ce tesson.

2) Fragment de moule de vase caréni. J)rangendorff 29 (pl. $1, n^{0} 2$ et pl. 4, no 2 ). Terre rose bien cuile, fine. A l'extérieur, traces de lissage sur le tour. en bandes étroites et parallieles. Le profil de la coupe élail assez evasé. La paroi, épaisse d'un cenlimilre porle un renforcement épais de $1 \mathrm{~cm}$. 4. Le diamilre du vase entier, aulant que l'on puisse le supposer, devail avoisiner 24 centimetres. . seul. le décor de la frise esl conservé (hauteur : 2 (m. 9). Il est composé uniquement de diclyl ras posés sur un petil molif circulaire et placés chacun dans un panneau reclangrulaire. délimilé par des lignes cordées verticales et des lignes pointillées horizontales. Le travail est minulieux el régulier. Le style de ce fragment et sa forme, comme ceux des autres moules et vases de cette trouvaille, se ratlachent directement a ceux des produits des fabriques de La Graufesenque, prés de Millau (Tarn) ${ }^{3}$. Cependant. le motif exact n'est donné ni par Knorr ni par llermet”; on en trouvera un trés proche chez le potier Incisorss, qui a travaillé a la fin du rìne de Tibere el au début de celui de Néron ${ }^{6}$. C'est aussi de cetle période ou plulòt Claude- Kéron, que nous daterions ce document, c'est-it-dire de celle qu'on a appelée la période de splendeur des fabriques rulhenes.

3) Fragment de moule de vase hémisphérique I)ragendorfl 37 (pl. 1, no 3 et pl. 4, no 3). Terre rose, plus apparentée ì celle du no 1 que du no 2 , peu cuite comme elle, assez mince : 7 millimetres. Le sommel des parois est asiez aigu aussi. et le renforcement a la mème épaisseur : 9 millimetres. - Le décor comprend une corbeille de godrons séparés par des liznes tremblées; au-dessus, une guirlande de larges feuilles imbriquées el stylisées. Enfin. une ligne de points el une pelite gorge. Il ne semble

(2) F. Oswal.d et F. I). Pryce, An Introduclion lo the Sludy of Terra Sigillala, pl. XXXII, no 19. K.xonk, Aislingen, pl. XVII, 4.

(3) Hквмкт, La Graufesenque, 1934, I I, pl. 1:.

(4) R. KNonR, Töpfer und Fabriken verzierter Terra-sigillala des ersten .Jahrhunderts.

(5) HI:RME:T, op. cil.

(6) R. K.хов, Töpfer..., pl. 40, no 20 . pas que nous ayons là un vase Iragendorlt 37 classique, ni pour la forme, ni pour le décor. En effet. j’ai relevé, au Musée de Périgueux, un frament de coupe lire d'un moule de la mime série que celui que nous étudions (pl. 1, lig. 3 bis. Au lieu de godrons, la panse est ornée de rinceaux, mais ils sont surmontés de la mime quirlande de feuilles. de la lignne pointillée el de la petite moulure. Ie rebord a élé monté verlicalement par le potier sur le tour, mais il ne porte point la livre demironde qui caractérise le vase Dragendorff $37^{7}$. Le decor de ce lesson no 3 est semblable, dans ses éléments el sa disposilion, a relui de la panse de cerlaines coupes arénées, tel celui que Hermet, reproduit pl. 108, no 2. Peul-on le dater de la même période, c'est-a-dire des Flaviens? Iu premier abord, cela parail normal, puisque les godrons séparés par des lignes tremblées sont une caractéristique chronologique suivant le mème auteur ${ }^{8}$. Cependant, il se pourrail que le vase que nous éludions fût un peu plus ancien, car le godron droil apparait tres tot (Claude) ${ }^{9}$ et parce que ce moule ressemble beaucoup par sa facture au no 1. Aussi proposerai-je de le placer plutòl sous Néron, en raison aussi du soin avec lequel le décor est tracé.

4) Fragment de moule de vase forme 1)ragendorff :37 (pl. 2. no t et pl. 4, no 4). Terre épaisse (1 cm. 2), rose, mème couleur que les autres fragments. - Au-dessus et au-dessous du décor, une ligne pointillée; de gauche à droite : trois panneaux, de haut en bas, frise de grlands, guirlande bi-foliée entre deux lignes pointillées, panneau de poinles de fleches. En allant vers la droile : grosise ligne verlicale cordée; grand médaillon a trois cercles entourant un élément slylisé. Iux 4 coins de la metope, 4 volutes. Les oves sont maladroilement disposées el le décor peu soigné : Ia Graufesenque ou Banassac, période de la décadence (Nerva-Trajan).

(7) Ph. DE Sindetzex et M. VAxDERHovex, La lerra sigillala a Tongres, p. 256 .

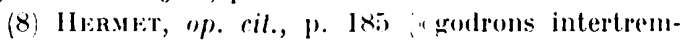
hles $"$;.

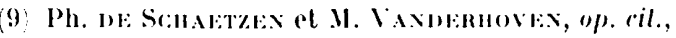
p. 116. 


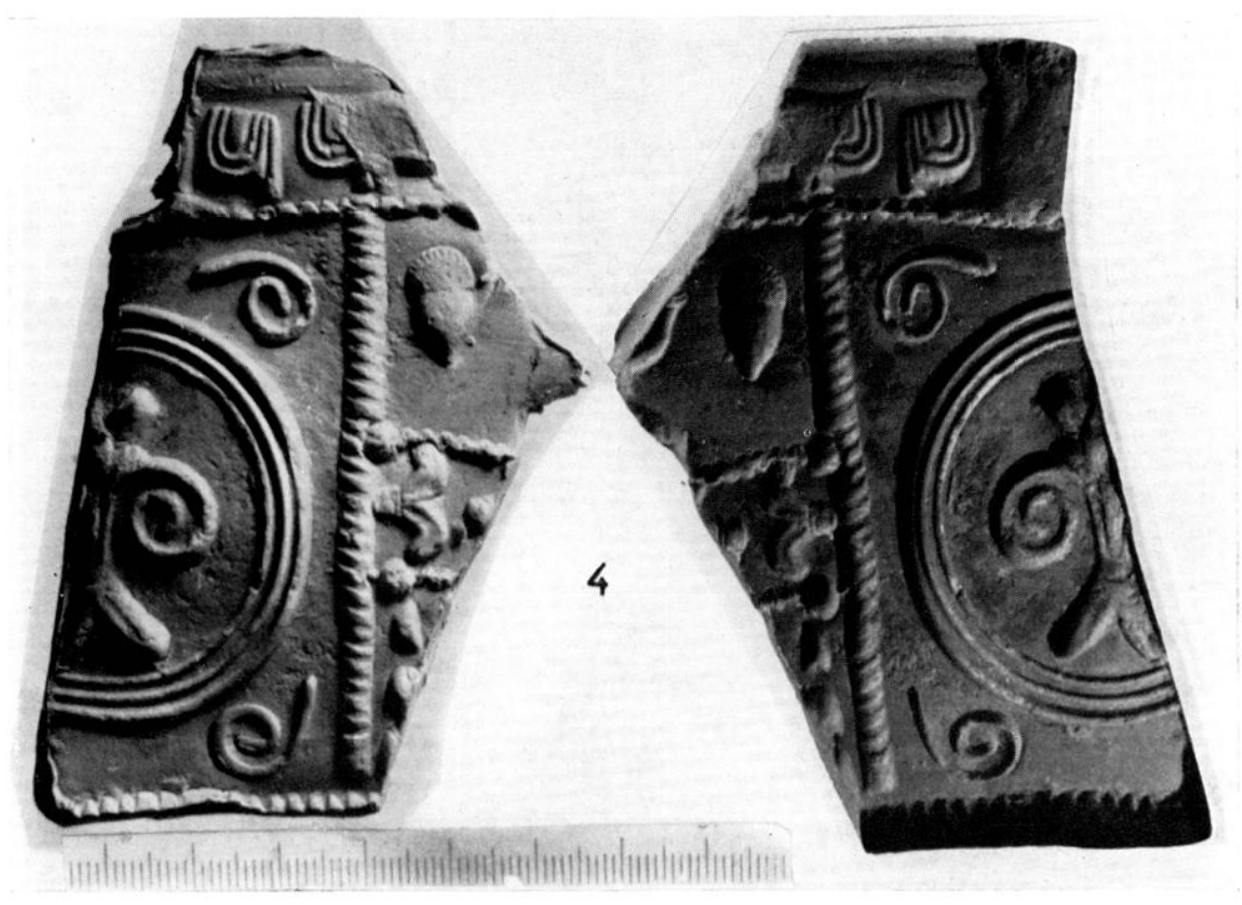

Planche :. - X゙" 4, moule et moulage à la plastiline du fragment $n^{\circ} 4$. (Photo II. Vertel).

\section{Fragmenls de rases.}

Parmi les 11 fragments de vases recueillis par M. Colles. 10 sonl de la forme Inragendorff 37 , el 1 de la forme linorr 78 ithermel 10: Il reste deux fonds. mais aucun ne porte de signalure. Vous les arons numérotés à la suile des moules.

\section{Forme 3i:}

5) Terre rose, surface rouge, mate. Epaisseur de la terre : 8 millimedres en bas. 5) en haut (pl. 3. no 5). Lu bas du décor, guimande. ligne pointillée; au-dessus, mélopes séparées par les mêmes lignes pointillées : de qauche a droite: a) pointes de fleches. b) feuilles ef tiges recourbées superposées, croix de saintAndré avec feuilles.... c) ? d) pointes de flèches. Période de la décadence. meilleur slyle que le moule no 4 : Iomitien-Trajan.

(6) Fond de vase paraissant tiré du même moule (pl. 3, no 6 et pl. 4, no 6 ) : a été trop cuit ; couleur violette et sable fondu collé à la pâte; épaisseur 5 millimètres au plus mince, 1 centimètre à côté en pied; Domitien-Trajan.

7) Haul de vase (pl. 3, no 7 et pl. 4, no 7 ): le décor est semblable, mais la cuirlande, au lieu d'avoir $1 \mathrm{~cm}$. ) de large n'a qu'un centimetre au-dessous, ligne pointillee. DomitienTrajan.
8) Fragment de bol netlement plus petit (pl. 3. no 8 ) : épaisseur maxima : 8 millimel res. minima 7 millimelres. Ligne de pelits médailons à double cercle contenant un décor de remplissage analogue a celui de la zone supérieure, qui est séparée par une ligne pointillée. Celle-ci est divisée en mélopes par de doubles lignes verticales tracées maladroitement a la roulelle. Il ne reste qu'un arand médaillon a trois cercles lrés écrasés contenant un élement à qualre fevilles. Période de la décadence: vers 100 apres .J.-C.

9) Fragment de petit bol 37 (pl. 3. no 9 ed pl. 4. no 9: : épaisseur : 6 millimelres: le diametre ne dépassail pas 16 centimetres: probablement du même potier que le no 4 : doubles lignes pointillées séparant les mélopes. pointes de fleche isoleses servant de motif de remplissage : de wauche a droile : salyre marchant vers la droite (oswald no i) Hermet nos 80-81, période flaviennei ; médaillon à double cercle contenant une protome d'animal (?). I)écor tris écrasé. Période de la décadence ; vers 100 apres .J.-C.

10) Fragment de panse (pl. 3. no 10) : oves. ligne pointillée, suite déléments décoralifs ? . guirlande. Période de la décadence.

11 Fragment de panse (pl. 3, no 11 : oves. 


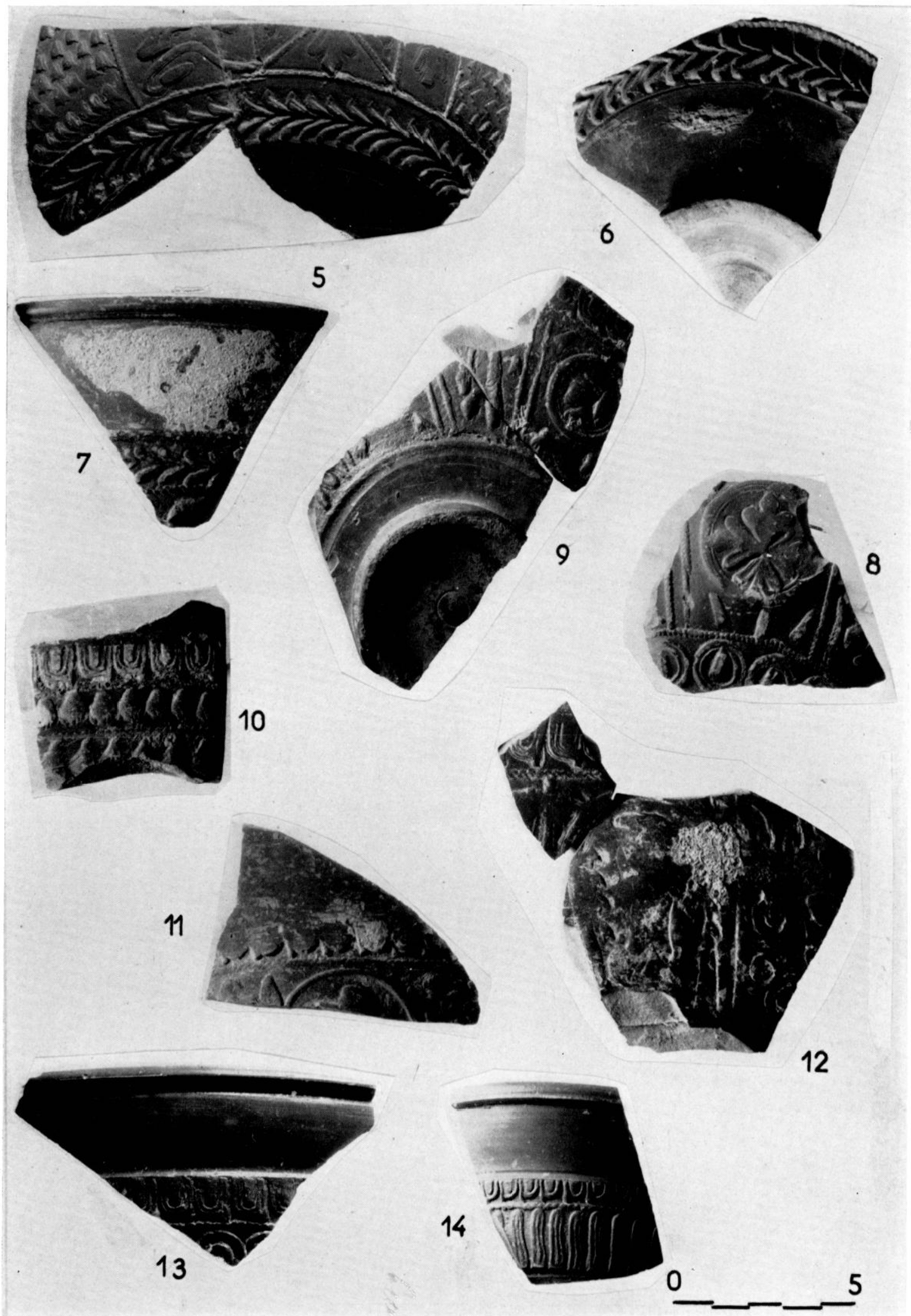

Planche 3. Tessons de vases trouvés avec les moules dans la source de chauvignac les numíros correspondent a ceux de l'ilude. (I'holo II. lertel). 
MOLLES DE SIGILLEE A BARZAN
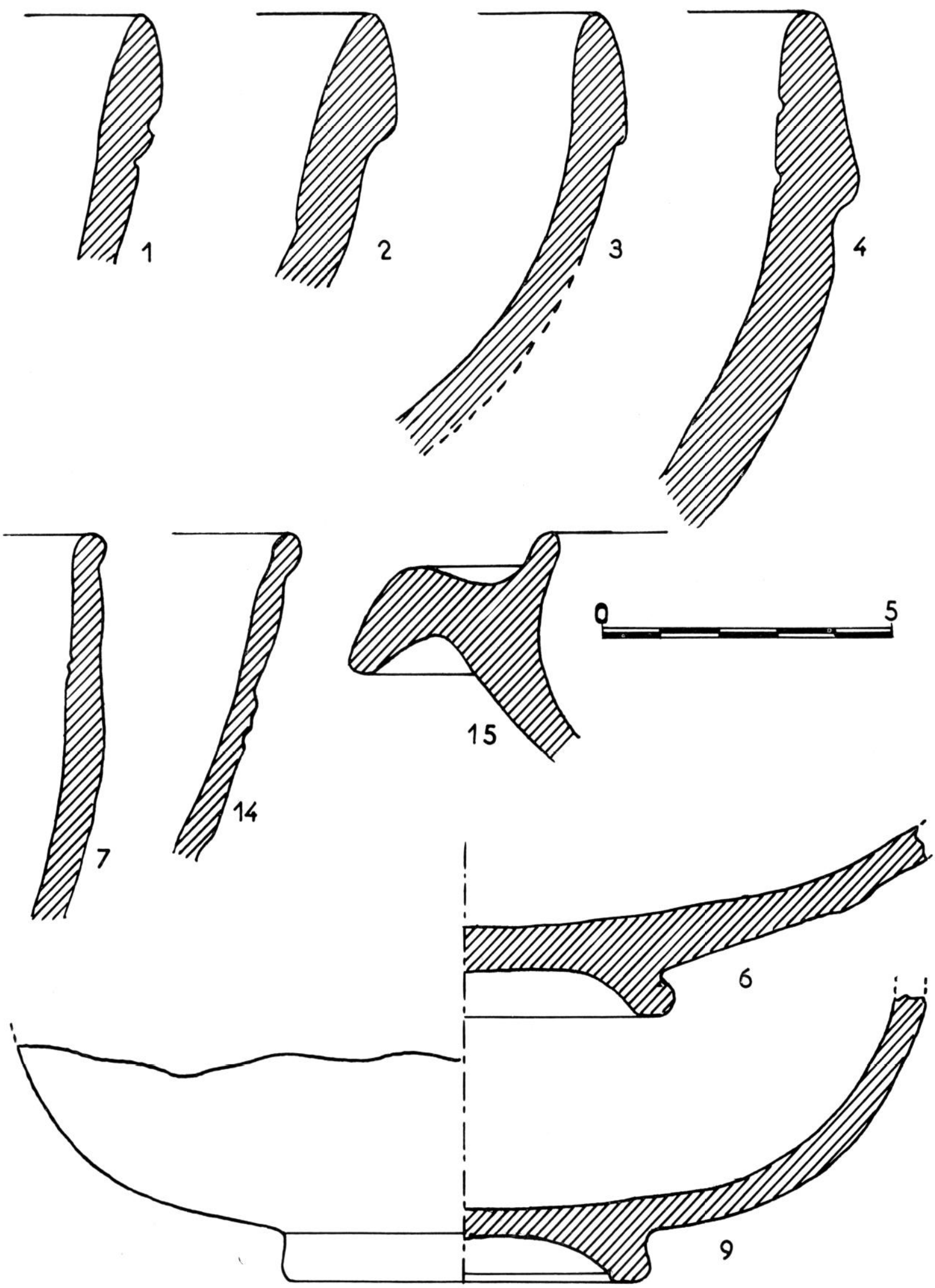

Planche 4. -- Profils des moules nos $1,2,3,4$ et profil des tessons les plus complets, $n^{\text {ns }} 6,7,9,14,15$ (I)essins G. Pic el II. Ve'lel). 
lige de rinceau ou médaillon au-dessous; probablement une aile; de chaque còlé, pelil personnage courant vers la gauche, les bras repliés, le coude gauche en arriere. le droit an avant.

12) Fragment de pantio (pl. 3, no 12) : de gauche a droite, liane a la biche sous une arcalure; avere un pelil poincon-matriee. le polier a figure des fenillages de chatpe cole de la deesse cespecess de pointes de fliodnes superposés ; mème grosse ligne verlicale cordec que sur le monle no 1 ; melope coupere : a) en haul, vépéalions styliseres (?) juxtaposées, en bas, séparé par une ligne pointillée. élément rayonnant fait de volutes analogues à celles du moule no $n^{0}$, maladroilement placées autour d'un motif qui a disparu. Le lout est Iris écrasé au démoulage. Periode de la décadence comme les lesions précédents.

13) Haut de vase (pl. 3. no 13) : oves. ligne pointillée. volutes rappelant celles du moule no 4 : période de la déadence.

1.1) Fragment de potiche. Hermet. forme 10: knorr, forme is (pl. 3. fig. 14 et pl. 4, fig. 14) : diamelre probable. 12 aentimelres. Décor : oves, ligne pointillée. godrons tres légerement on S; of. Hermel, pl. 92) no 1 palissades strigillées). Vespasien-Domitien.

le vernis de ces lessons est divers : tres brillant sur le no 12 ef le no 13 , beaucoup moins sur les aulres, el toul a fail mal sur les nos 10 et 6 .

Polerie lisse. In seul fragment de mortier a rebord tombant, surface claire (coupe : pl. 1. fig. 15). Date : Domitien-Trajan.

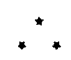

Ces fragments de moules of de vases sigilles sont probablement, comme les monnaies, des ex-voto lancés dans la source par les fideles. Il est vaisemblable que parmi les dédicants se trouvaient les potiers eux-mèmes. In eflet on peut admelle que les moules relroures étaient les instruments de travail d'une fabrique voisine.

D'après une enquète faile sur place, les terrains qui entourent la source ne contiennent pas d'argile. Mais il ne faut pas aller bien loin pour en trouver. A quelques kilomelres de lá, sur la mème commune de Barzan, au hameau aujourd'hui abandonné de Boën, on trouve, sous le sol arable sablonneux, des couches d'aroile que les gens du pays appellent du nom significalif de "lerre a pots". Ies officines se lrouvaient-elles li-bas ou plus pres de l'eau? Je n'ai pas eu le lemps de faire de longues prospections sur place. Guant a la région où surat la source, elle est actuellement, soit comverte d'une végétalion luxuriante de joncs, de peupliers, ele, soil oceupee par des champs recouverts d'un limon épais, où je n'ai rien troure. On peut cependant esperer siluer un jour les alceliers des poliers. car la région est ou cultivere ou en friche. mais il n'y a point de cess erandes installations urbaines ou industrielles si néfastes aux wisements antiques. L'élude de la céramique trouvée dans les fouilles du temple dit le "Moulin du Fà permettrait peut-etre deja de préciser si celle. fabrique avait un slyle de décoration particulier.

Il est deja imporlanl de savoir que le porl actil des Santons qu'élail le Fà de Talmont possedail une fabrique de sigillée dont le fonctionnement est altesté de Claude à Trajan, el s'elend peutètre sur une période plus longrue. Quelle fut l'origine de cel atelier? Il se peul qu'une parlie de l'exportation des oflicines de La Graufesenque vers la Brebanne ot l'Angleterre soil passé par la. Eille se serail jointe au grand courant commercial qui partail de la Médilerranée pour aller aux Iles Britanniques, dont la roule la plus courle, terrestre d'abord, marilime ensuite, passait par le seuil de Naurouze, la Gironde et suivait la còle par cabolage. Il ne serait pas étonnant qu'une escale el un dépòt de vases ruthénes ait existé a Talmont, et qu'il s'y soit adjoint assez rapidement une fabrique de sigillée, soil par un apport de moules de la maison mère. soit par l'arrivée d'ouvriers spécialisés. soit par les deux, a partir du moment que l'on a appelé la période de splendeur, ou l'essor des industries ruthenes chail à son apogée.

\section{Hugues Virtet.}

10 Les produits de cess fahriefues-shecursales diffirent de ceux des maisons meres par quelpues details :

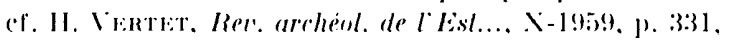
11. : : lilla gallo-romaine ì lélines (Lerdogne), dans ogam. Xlll-1961, l. 557; mimes ohservations sur les petites fabriques voisines des qrands centres : Jo/ficine de Toulon-sur-.1llier, dans Rei crelariae Faulorum . Irla. II, 1959 . 